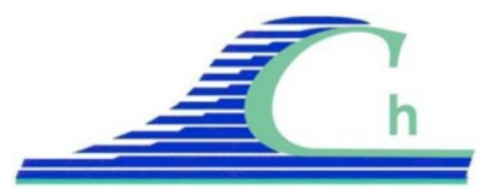

XII ${ }^{\text {ìmes }}$ Journées Nationales Génie Côtier - Génie Civil

Cherbourg, 12-14 juin 2012

DOI:10.5150/jngcgc.2012.084-P @ Editions Paralia CFL

disponible en ligne - http://www.paralia.fr - available online

\title{
Influence de la remontée du niveau de la mer sur les franchissements des ouvrages maritimes selon trois approches
}

\section{Guirec PREVOT ${ }^{1}$, Xavier KERGADALLAN ${ }^{2}$, $^{\text {Philippe SERGENT }}{ }^{1}$}

1. Centre d'Etudes Techniques Maritimes et Fluviales, 2, Boulevard Gambetta, 60200 Compiègne, France.guirec.prevot@developpement-durable.gouv.fr philippe.sergent@developpement-durable.gouv.fr

2. Centre d'Etudes Techniques Maritimes et Fluviales, Technopôle Brest Iroise, 29280 Plouzané, France. xavier.kergadallan@developpement-durable.gouv.fr

\section{Résumé :}

L'influence du changement climatique sur les infrastructures maritimes (digues de protection des ports et ouvrages de protection du littoral) est importante en termes de franchissement. Dans cet article, trois méthodes sont proposées pour estimer cette sensibilité. Le but est de déterminer par trois approches la rehausse nécessaire des digues pour conserver la même performance hydraulique qu'actuellement.

La première méthode utilisée est une méthode analytique. La propagation de houle est réalisée à l'aide de la méthode de Goda (GODA, 2000) et des formules du TAW (TAW, 2002). Cette approche conduit à une proposition de formule simplifiée reliant la rehausse de la digue à l'augmentation du niveau de la mer. Une méthode numérique est ensuite utilisée pour vérifier les résultats de l'approche analytique et, donc la formule proposée. Puis, pour finir, une méthode statistique est mise en œuvre pour estimer l'évolution de la cote de la crête de la digue. Cette méthode est assez originale car le dimensionnement de la structure n'est plus effectuée à partir de la cause (houle et niveau) mais de la conséquence (débit franchissant). Cette dernière méthode modère les résultats des deux premières approches. Néanmoins, l'ensemble de ces approches montre que le changement climatique et sa conséquence principale (le remontée du niveau moyen de la mer), induira des adaptions d'ouvrages.

Mots-clés : Franchissements - Digues - Protections côtières - Augmentation du niveau moyen des mers - Approche Statistique - Approche Analytique - Approche Numérique

\section{Introduction}

Le changement climatique pourrait induire des effets non négligeables sur les structures de protection : l'intensification des tempêtes aux larges (et donc des houles) et l'augmentation du niveau moyen des mers. Il est donc logique de s'intéresser aux conséquences de la modification de ces deux paramètres sur les franchissements. Pour cela trois approches sont développées (analytique, numérique et statistique). Les franchissements sont calculés par des formules très utilisées et bien connues (TAW, 2002). 
La méthode de Goda (GODA, 2000) est utilisée dans la méthode analytique afin de propager la houle du large à la côte. Quelques travaux, sur l'influence du changement climatique sur les ouvrages ont déjà été réalisés dans le cadre du projet Discobole (LEBRETON \& TRMAL, 2009). Cette étude montrait l'influence du changement climatique sur un ouvrage en termes de franchissement et de stabilité à l'aide d'un outil numérique (REEF, 2000) pour la propagation de la houle. Dans cette étude, l'approche numérique reprendra cette méthode afin de vérifier les résultats de la méthode analytique. L'approche statistique quant à elle, est réalisée à l'aide d'une méthode de Monte Carlo et se base sur les probabilités conjointes (Houle, Niveau) sur lesquels certains auteurs ont déjà travaillés. DEFRA/Environment Agency, résume en 2005 (DEFRA, 2005), l'ensemble de ces travaux et propose un guide méthodologique sur les méthodes de probabilités conjointes pour le domaine de l'aménagement côtier. Une base de données (Houle, Niveau) a donc pu être réalisée, puis utilisée pour déterminer les franchissements sur des ouvrages particuliers (deux sites d'études St Malo et Deauville).

Cet article compare donc l'influence du changement climatique selon ces différentes approches. Le but est de déterminer pour chaque méthode la rehausse de la digue nécessaire afin de conserver les mêmes performances hydrauliques, i.e. le même débit franchissant. L'approche analytique conduit à proposer une formule nouvelle, reliant l'augmentation du niveau de la mer à la rehausse de la digue. Cette formule est ensuite vérifiée par la méthode numérique.

La méthode statistique est quant à elle originale dans le sens où elle utilise les méthodes de probabilités conjointes pour évaluer l'influence du changement climatique sur l'évolution de la cote d'arase des protections.

Les différentes méthodes seront d'abord présentées, puis seront utilisées pour estimer le renforcement de l'ouvrage nécessaire (augmentation de la crête de l'ouvrage). Enfin, l'ensemble des résultats seront comparés.

\section{Principe des trois approches}

\subsection{Paramètres et formules de franchissement}

Les infrastructures maritimes sont classiquement dimensionnées grâce aux houles incidentes de projet et aux niveaux d'eau de projet sans tenir compte d'une corrélation entre ces deux variables.

Dans le but d'étudier les impacts et conséquences du changement climatique sur le franchissement les formules du TAW (TAW, 2002) sont utilisées : elles relient la revanche de l'ouvrage $\left(\mathrm{R}_{\mathrm{c}}\right)$ (différence entre les cotes de crête et niveau d'eau de dimensionnement) aux caractéristiques de la houle de dimensionnement (hauteur $\mathrm{H}_{\mathrm{m} 0}$ et période via le paramètre de déferlement $\xi_{\mathrm{m}-1,0}$ ) en tenant compte de la pente de l'ouvrage $(\alpha)$, obliquité de la houle $\left(\gamma_{\beta}\right)$ et du type de revêtement du talus $\left(\gamma_{\mathrm{f}}\right)$. 


\section{XII ${ }^{\text {èmes }}$ Journées Nationales Génie Côtier - Génie Civil \\ Cherbourg, 12-14 juin 2012}

Paramètre de déferlement $\xi_{\mathrm{m}-1,0}$

$$
\xi_{m-1,0}=\tan \alpha \frac{\sqrt{\left(g T_{m-1,0}^{2}\right)}}{\sqrt{\left(2 \pi H_{m 0}\right)}}
$$

Pour $\xi_{\mathrm{m}-1,0}>7$ (en faible profondeur d'eau)

$$
\begin{gathered}
q / \sqrt{g H_{m 0}{ }^{3}}=0.21 \exp \left(\frac{-R_{c}}{\gamma_{f} \gamma_{\beta} H_{m 0}\left(0.33+0.022 \xi_{m-1,0}\right)}\right) \\
q / \sqrt{g H_{m 0}{ }^{3}}=C \exp \left(-D \frac{R_{c}}{H_{m 0}} \frac{1}{\gamma_{f} \gamma_{\beta}}\right) \\
q / \sqrt{g H_{m 0}{ }^{3}}=\frac{A}{\sqrt{\tan \alpha}} \gamma_{b} \xi_{m-1,0} \exp \left(-B \frac{R_{c}}{H_{m 0}} \frac{1}{\xi_{m-1,0} \gamma_{b} \gamma_{f} \gamma_{\beta}}\right)
\end{gathered}
$$$$
\text { Pour : } \xi_{\mathrm{m}-1,0}<2
$$

Figure 1. Formules de franchissement (TAW, 2002) et leurs domaines de validité.

\subsection{Approche analytique}

La méthode analytique est développée autour de la formule de Goda (GODA, 2000) afin d'analyser les effets du changement climatique sur la houle à la côte. Cette formule a pour paramètres principaux, la hauteur de houle significative au large $\left(\mathrm{H}_{0}\right)$ et la profondeur d'eau au pied de l'ouvrage (h). Trois termes interviennent: un terme correspondant au shoaling $\left(\mathrm{K}_{\mathrm{s}} \mathrm{H}_{0}\right)$, un autre pour le déferlement $\left(\beta_{0} \mathrm{H}_{0}+\beta_{1} \mathrm{~h}\right)$ et le dernier permettant de faire le lien entre la phase de shoaling et de déferlement $\left(\beta_{\max } \mathrm{H}_{0}\right)$.

$H_{1 / 3}=\min \left[\left(\beta_{0} H_{0}+\beta_{1} h\right), \beta_{\max } H_{0,} K_{s} H_{0}\right]$

Dans nos hypothèses, le changement climatique a deux effets principaux :

- L'augmentation du niveau de la mer

- Et, une variation de la hauteur houle au large

Les deux effets modifient le climat de houle à la côte : la variation de la houle est notée $\Delta$ Hs. Dans la zone de déferlement, le premier terme de la formule (4) prévaut.

$$
H_{s}=\beta_{0} H_{0}+\beta_{1} h
$$

L'étude de la variation conduit à la formule suivante :

$$
\Delta H_{s}=\mu \beta_{0} \Delta H_{0}+\beta_{1} \Delta h
$$

où $\mu$ est un coefficient issu de la dérivation

Puis, du fait de la prépondérance du terme lié à la profondeur d'eau, la formule simple suivante peut être retenue (valable en zone de déferlement et pour une pente des fonds faible devant l'ouvrage) :

$$
\Delta H_{s} \approx \Delta H_{m 0} \approx 0,55 \Delta h
$$

\subsection{Approche numérique}

Le logiciel REEF 2000 (BENOIT, 2006) a été utilisé afin de vérifier numériquement le développement effectué plus haut. Pour cela, plusieurs cas de houle ( $\mathrm{Hm} 0, \mathrm{Tp})$ au large ont été propagés $\{(4 \mathrm{~m}, 6 \mathrm{~s}),(8 \mathrm{~m}, 10 \mathrm{~s})$ et $(12 \mathrm{~m}, 14 \mathrm{~s})\}$ et sur différentes pentes $(1 \%$, $5 \%, 10 \%)$.

Les résultats obtenus montrent que la simplification effectuée (en 2.1) est bonne pour de faible pente. Nous utiliserons donc ce résultat par la suite. 


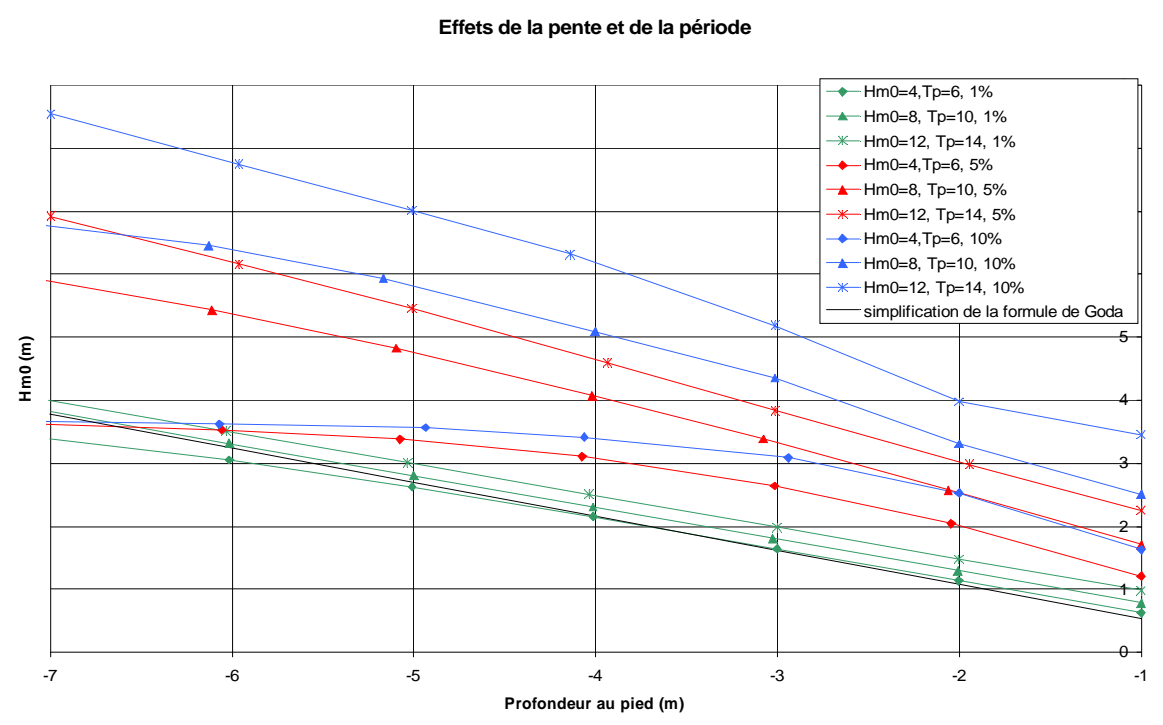

Figure 2. Confrontation des résultats en hauteur significative spectrale, en fonction de la profondeur au pied de l'ouvrage, avec REEF 2000 et la formule simplifiée.

\subsection{Approche statistique}

Le principe de cette méthode, inspirée par Hawkes (HAWKES, 2002), repose sur la modélisation de la dépendance vague/surcote à partir d'un échantillon d'observation simultanée de vagues et de surcotes, afin de générer, par simulation de Monte Carlo, un échantillon de données fictives simultanées de vagues et de surcotes sur une durée importante. S'intéressant à des franchissements de période de retour plus ou moins importante, la durée de simulation est portée à 10000 ans.

Cette méthode nécessite dans un premier temps une analyse distincte des probabilités d'occurrence de hauteur de vague de pleine mer (hauteur significative maximale atteinte entre deux pleines mers consécutives) et des surcotes de pleine mer (différence entre le niveau maximum observé et prédit au voisinage du moment de pleine mer). Dans un second temps la modélisation de la dépendance vague/surcote est effectuée, via un changement de variable (transformation en distribution normale centrée), dans un espace de travail normalisé (fonction normale bivariée).

Une fois la base de données de 10000 ans d'états de mer générée soit 7060000 couples (houle, niveau), l'ensemble est propagé à la côte, puis les franchissements calculés. Une coupe type a été choisie et les données de niveau et de houle ont été prises sur St Malo. Cet ouvrage type est dimensionné à partir des formules précédemment citées avec un débit $\mathrm{q}=1 \times 10^{-3} \mathrm{~m}^{3} / \mathrm{s} / \mathrm{ml}$, pour la houle annuelle $(\mathrm{Hs}=4.05 \mathrm{~m})$ et le niveau annuel $(\mathrm{N}=13.33 \mathrm{~m})$. La période de retour d'un tel débit est d'environ 12 ans sur l'ouvrage.

Le but de cette méthode est aussi de déterminer la rehausse de la digue nécessaire pour conserver les mêmes caractéristiques de débit en quantité (ie $\mathrm{q}=1 \times 10^{-3} \mathrm{~m}^{3} / \mathrm{s} / \mathrm{ml}$ ) et en période de retour (i.e. 12 ans). 


\section{XII ${ }^{\text {èmes }}$ Journées Nationales Génie Côtier - Génie Civil \\ Cherbourg, 12-14 juin 2012}

Plusieurs scénarios sont pris concernant l'évolution des niveaux d'eau à l'horizon 2100, en lien avec les estimations de l'ONERC (ONERC, 2010) :

- $+40 \mathrm{~cm}$, hypothèse optimiste ;

- $+60 \mathrm{~cm}$, hypothèse pessimiste ;

- $+100 \mathrm{~cm}$, hypothèse extrême.

\section{Résultats des trois approches}

3.1 Formules d'estimations de la cote de la crête de la digue.

Les résultats de l'étude analytique sont réutilisés dans les formules de franchissement. La variation de la cote de crête $(\Delta D)$ entre l'état final (avec élévation du niveau d'eau) et l'état actuel, avec un développement limité à l'ordre 1, conduit à la formule simple suivante pour des débits faibles (i.e $q<5 \times 10^{-2} \mathrm{~m}^{3} / \mathrm{ml} / \mathrm{s}$ ).

$$
\Delta D=\left[1-\gamma_{f} \gamma_{B} 0,55 *(0,33+0,022 \xi) \ln q\right] \Delta h
$$

3.2 Comparaison de la formule simplifiée avec l'emploi du modèle numérique

Pour comparer les résultats obtenus par la simplification, ceux-ci ont été confrontés aux résultats obtenus par l'emploi classique de la méthode de dimensionnement : calculs des houles à la côte par un modèle numérique et utilisation des formules de franchissements.

La formule simplifiée proposée donne une erreur moyenne de 7\% sur l'ensemble des cas étudiés (erreur maximum de 12\%) par rapport à la méthode sans simplification.

On pourra retenir l'ordre de grandeur suivant pour cette méthode : pour conserver la performance hydraulique sur des ouvrages en enrochements, il faut en fonction des débits, rehausser l'ouvrage de $1.50 \mathrm{~m}$ à $2.50 \mathrm{~m}$ pour une élévation du niveau d'un mètre.

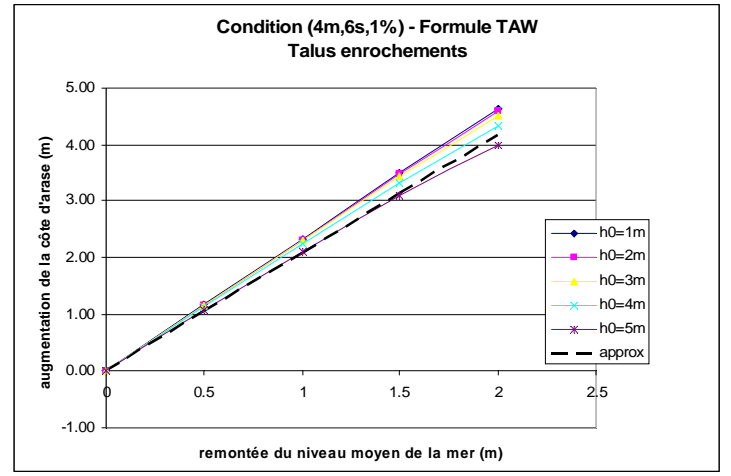

(3a)

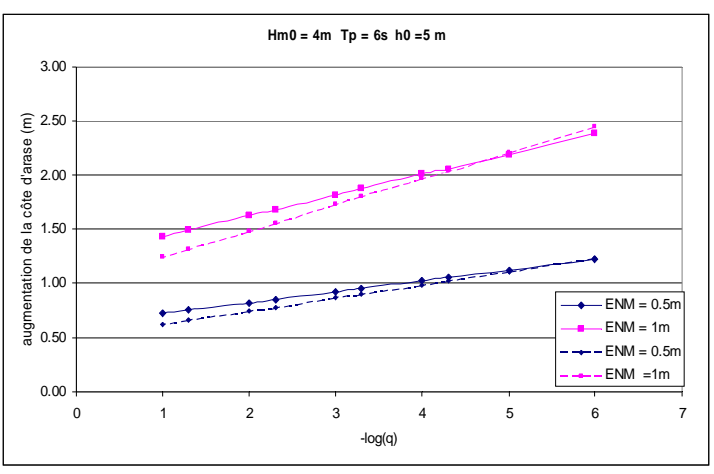

$(3 b)$

Figure 3. Rehausse de l'ouvrage en fonction de l'élévation du niveau de la mer (ENM) croisée avec la profondeur initiale au pied de l'ouvrage $\left(h_{0}\right)(3 a)$ et le débit d'étude $(3 b)$. Les courbes en pointillés correspondent à l'approximation. 


\subsection{Résultats de l'analyse statistique}

3.3.1 Résultats obtenus sur un ouvrage type à St Malo avec un débit de $1 \times 10^{-3} \mathrm{~m}^{3} / \mathrm{s} / \mathrm{ml}$ Les graphiques suivants sont obtenus (figure 4). On trouve en ordonnées les hauteurs de houle au large $\left(\mathrm{H}_{\mathrm{s}}\right)$ et en abscisses les niveaux d'eau ( $\mathrm{N}=$ marée + surcotes). Les courbes concaves représentent les courbes d'isoprobabilité des couples $\left(\mathrm{H}_{\mathrm{s}}, \mathrm{N}\right)$. C'est à dire, tous les couples $\left(\mathrm{H}_{\mathrm{s}}, \mathrm{N}\right)$ situés sur une courbe ont la même période de retour. Les courbes convexes représentent les courbes d'iso-arase (déterminées à partir des formules du TAW). C'est-à-dire, l'ensemble des couples situées sur une iso-arase occasionnent un débit de $1 \times 10^{-3} \mathrm{~m}^{3} / \mathrm{s} / \mathrm{ml}$. Le but de l'exercice est donc de déterminer la courbe dont le débit a une période de retour de 12 ans. Les points de couleurs représentent les 7060000 conditions (couples) : les roses correspondant à des conditions occasionnant des débits supérieurs au débit dodécennal.

Une partie des points bleus (partie en bas à gauche) ne figure pas. Les conditions correspondant à ces points n'ont pas été calculées (conditions peu sévères, ne contribuant pas aux résultats) permettant de réduire le temps de calcul.

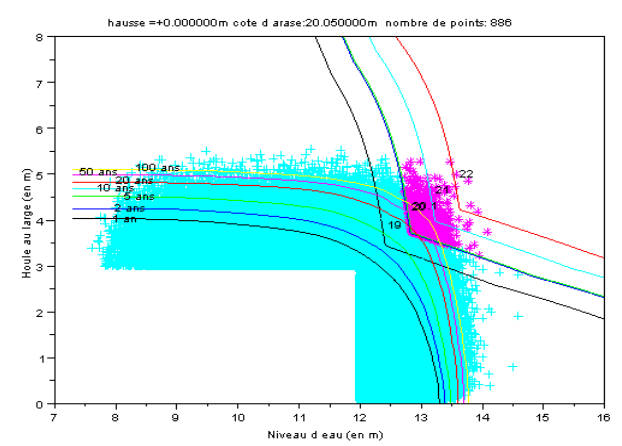

a) situation actuelle

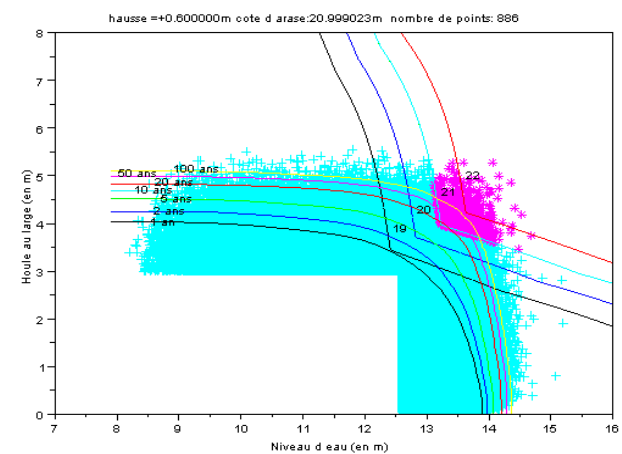

c) élévation du niveau de la mer : $+60 \mathrm{~cm}$

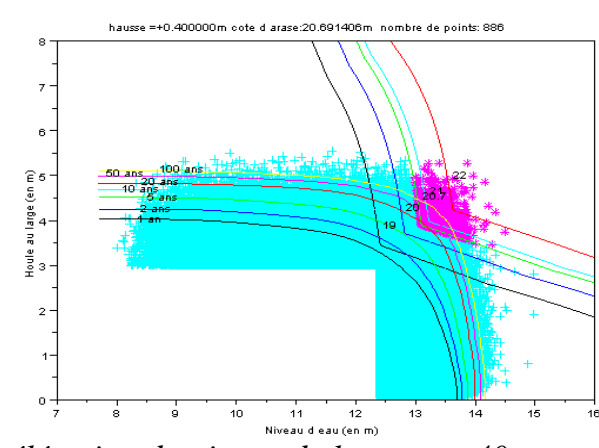

b) élévation du niveau de la mer : $+40 \mathrm{~cm}$

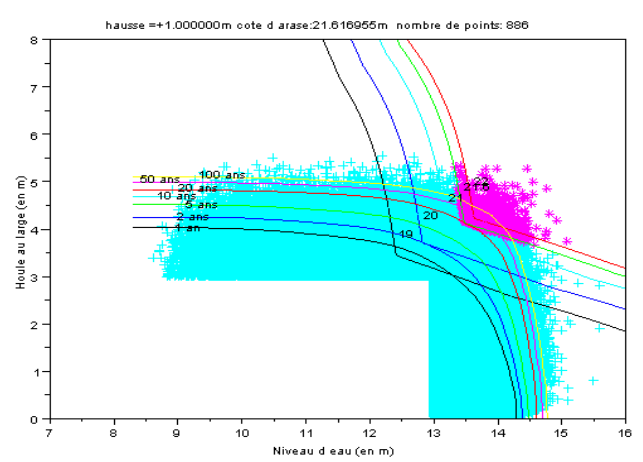

d) élévation du niveau de la mer : $+100 \mathrm{~cm}$

Figure 4. Évaluation des côtes d'arase en fonction de l'élévation du niveau de la mer. 


\section{XII ${ }^{\text {èmes }}$ Journées Nationales Génie Côtier - Génie Civil \\ Cherbourg, 12-14 juin 2012}

3.3.2 Résultats obtenus sur un ouvrage réel à Deauville avec un débit de $5 \times 10^{-2} \mathrm{~m}^{3} / \mathrm{s} / \mathrm{ml}$ La même méthode est employée sur ce site mais avec un débit d'étude plus important que celui de St Malo. Les résultats sont indiqués dans le tableau ci-dessous (figure 6).

\begin{tabular}{|c|c|c|c|c|}
\hline Ouvrage type de St Malo $-q=1 \times 10^{-3} \mathrm{~m}^{3} / \mathrm{s} / \mathrm{ml}$ \\
\hline Augmentation du niveau d'eau (cm) & 0 & 40 & 60 & 100 \\
\hline Augmentation de la cote d'arase de la digue (cm) & 0 & 70 & 100 & 161 \\
\hline
\end{tabular}

Figure 5. Évolution des cotes d'arase pour St Malo.

\begin{tabular}{|c|c|c|c|c|}
\hline Digue de Deauville $-q=5 \times 10^{-2} \mathrm{~m}^{3} / \mathrm{s} / \mathrm{ml}$ \\
\hline Augmentation du niveau d'eau $(\mathrm{cm})$ & 0 & 40 & 60 & 100 \\
\hline Augmentation de la cote d'arase de la digue $(\mathrm{cm})$ & 0 & 57 & 85 & 140 \\
\hline
\end{tabular}

Figure 6: Évolution des cotes d'arase pour Deauville.

\section{Confrontation des résultats de l'étude analytique et de l'étude numérique}

Pour des ouvrages en enrochements, l'étude analytique montre que pour une élévation d'un mètre du niveau de dimensionnement, la rehausse de l'ouvrage est comprise entre $1,50 \mathrm{~m}$ et 2,50 m en fonction des débits d'étude : pour un débit de $5 \times 10^{-2} \mathrm{~m}^{3} / \mathrm{s} / \mathrm{ml}$, la rehausse est comprise entre $1.65 \mathrm{~m}$ et $1.85 \mathrm{~m}$, alors que pour un débit de $1 \times 10^{-3} \mathrm{~m}^{3} / \mathrm{s} / \mathrm{ml}$ cette rehausse est comprise entre $1.80 \mathrm{~m}$ et $2.10 \mathrm{~m}$.

Les résultats de l'analyse statistique modèrent donc ces résultats car la rehausse prévue est de $1.40 \mathrm{~m}$ (Deauville) et $1.61 \mathrm{~m}$ (St Malo) respectivement pour $5 \times 10^{-2}$ et $1 \times 10^{-3} \mathrm{~m}^{3} / \mathrm{s} / \mathrm{ml}$.

Cette différence vient du fait que dans l'analyse statistique, l'ensemble des conditions de houle sont prises en compte alors que dans l'étude analytique seulement les cas de houles déferlées sont étudiés.

En outre, par lecture graphique, en mesurant la distance entre les lignes d'iso-arase (figure 4), les résultats de l'étude analytique peuvent être retrouvés. On obtient donc pour Deauville, une rehausse de $1.74 \mathrm{~m}$ et pour St Malo de $2.10 \mathrm{~m}$, pour une élévation du niveau d'eau d'un mètre. Ces résultats sont concordants avec ceux cités juste avant.

\section{Conclusion}

L'étude permet donc d'aboutir à plusieurs résultats en terme de rehausse nécessaire pour conserver la même performance hydraulique des ouvrages. Les exemples cités et comparés sont des ouvrages à talus en enrochements (mais les résultats sont transposables à d'autres talus via modifications des coefficients).

Pour des ouvrages implantés en zone de déferlement et sur des faibles pentes, une formule simplifiée est proposée pour estimer la rehausse des ouvrages à l'élévation du niveau de la mer. Cette formule est confrontée aux résultats obtenus par une méthode de dimensionnement classique (propagation de la houle par un outil numérique et application des formules de franchissement). Les résultats montrent que les 
franchissements sont très sensibles à l'augmentation du niveau moyen de la mer et que pour, par exemple, un mètre d'augmentation du niveau de la mer, les ouvrages doivent être rehaussés de $1.50 \mathrm{~m}$ à $2.50 \mathrm{~m}$ en fonction des débits critiques exigés.

La méthode statistique est originale et basée sur l'étude de la concomitance de la houle et des niveaux. Les résultats obtenus par cette méthode modèrent les précédents résultats analytiques, car intègrent toutes les conditions de houles.

L'approche statistique apporte une nouvelle vision dans le dimensionnement puisque la cote d'arase de la digue est déterminée à l'aide du débit franchissant et de sa période de retour. Cette approche est plus concrète pour le maître d'ouvrage, car la structure est dimensionnée par la conséquence (débit) et non plus par les causes (houles et niveaux). Ce travail a été réalisé dans le cadre du projet SAO POLO (Stratégies d'Adaptation des Ouvrages de Protection marine ou des modes d'Occupation du Littoral vis-à-vis de la montée du niveau des mers et des Océans). La méthode statistique a été utilisée dans le cadre d'une étude économique sur le territoire de l'agglomération havraise et pour la comparaison des différentes stratégies d'adaptation (NDIAYE, 2012), toujours dans le cadre du projet SAO POLO.

\section{Références bibliographiques}

BENOIT M. (2006). Notice d'utilisation du logiciel REEF 2000 version 6. EDF Chatou, $29 \mathrm{p}$.

DEFRA (2005). Use of joint probability methods in flood management - A guide to best practice. R\&D Technical Report FD2308/TR2 - DEFRA/Environment Agency, 67 p.

GODA Y. (2000). Random seas and design of maritime structure, PL-F Liu (ed), Advanced Series on Ocean Engineering, Vol. 15, World Scientific,Singapore, 444 p.

HAWKES P.J. (2002). The joint probability of waves and water levels in costal engineering design, Journal of hydraulic research, Vol. 40, $\mathrm{n}^{\circ} 3$, pp 241-251. doi:10.1080/00221680209499940

LEBRETON P., TRMAL C. (2009). Evaluation de l'impact du changement climatique sur le dimensionnement des digues à talus. La houille blanche, $\mathrm{n}^{\circ} 2$, pp 45-51. doi:10.1051/lhb/2009013

NDIAYE F., MOREL G., VOYNEAU N., PREVOT G. (2012). Méthode d'estimation des dommages économiques sur le territoire en cas de submersion marine et comparaison des stratégies d'adaptation - Application à la ville du Havre. UTC, Compiègne, $45 \mathrm{p}$.

ONERC (2010). Synthèse $n^{\circ} 2$, Prise en compte de l'élévation du niveau de la mer en vue de l'estimation des impacts du changement climatique et des mesures d'adaptation possibles, $6 \mathrm{p}$.

TAW (2002). Technisch rapport golfoploop en golfoverslag bij dijken (Technical report on wave run-up and wave overtopping at dikes - in Dutch). Technical Advisory Committee on Water Defences, Delft, $50 \mathrm{p}$. 